\title{
Randomized Controlled Trial on the EMDR Integrative Group Treatment Protocol for Ongoing Traumatic Stress with Adolescents and Young Adults Patients with Cancer
}

\author{
Amalia Osorio ${ }^{1}$, María Cristina Pérez ${ }^{1}$, Sofía Gabriela Tirado ${ }^{1}$, Ignacio Jarero ${ }^{2, \text { *, }}$ \\ Martha Givaudan ${ }^{2}$ \\ ${ }^{1}$ Department of Research, Ágape, Psycho-Oncology and Research Center, Puebla, Mexico \\ ${ }^{2}$ Department of Research, Mexican Association for Mental Health Support in Crisis, Mexico City, Mexico \\ Email address: \\ amaliaosoriovigil@hotmail.com(A. Osorio),mperezgrados@yahoo.com.mx (M.C. Pérez), tirado.gabriela@yahoo.com.mx (S. G. Tirado), \\ nacho@amamecrisis.com.mx (I. Jarero),martha@imifap.org.mx (M. Givaudan) \\ ${ }^{*}$ Corresponding author
}

To cite this article:

Amalia Osorio, María Cristina Pérez, Sofía Gabriela Tirado, Ignacio Jarero, Martha Givaudan. Randomized Controlled Trial on the EMDR Integrative Group Treatment Protocol for Ongoing Traumatic Stress with Adolescents and Young Adults Patients with Cancer. American Journal of Applied Psychology. Vol. 7, No. 4, 2018, pp. 50-56. doi: 10.11648/j.ajap.20180704.11

Received: October 21, 2018; Accepted: November 5, 2018; Published: November 30, 2018

\begin{abstract}
The aim of this randomized controlled trial was to evaluate the effectiveness of the EMDR-Integrative Group Treatment Protocol for Ongoing Traumatic Stress (EMDR-IGTP-OTS) in reducing posttraumatic stress disorder symptoms, depression and anxiety symptoms related to the diagnosis and treatment of cancer. Twenty-three adolescents and young adults (13 male and 10 female) with different types of cancer (breast, leukemia, lymphoma) and PTSD symptoms related to their diagnosis and cancer treatment met the inclusion criteria. Participants age ranged from 13 to 22 years old ( $\mathrm{M}=16.71$ years). Participant's time since diagnosis varied from 2006 to 2018. Participants in treatment $(\mathrm{N}=11)$ and no-treatment control $(\mathrm{N}=12)$ groups completed pre, post, and follow up measurements using the Posttraumatic Stress Disorder Checklist for DSM-5 (PCL5) and the Hospital Anxiety and Depression Scale (HADS). Data analysis by repeated measures ANOVA showed that the EMDR-IGTP-OTS was effective in significantly reducing symptoms of PTSD, anxiety, and depression, with symptoms maintained at 90-day follow-up and with large effect sizes (e.g., $\mathrm{d}=1.17$ ). A comparison of the treatment and no-treatment control groups showed significantly greater decreases for the treatment group on symptoms of PTSD, anxiety, and depression. This study suggests that EMDR-IGTP-OTS may be an efficient and effective way to address cancer-related PTSD, depressive, and anxious symptoms in adolescents and young adults.
\end{abstract}

Keywords: Eye Movement Desensitization and Reprocessing (EMDR), EMDR-IGTP-OTS, Posttraumatic Stress Disorder (PTSD), Anxiety, Depression, Adolescents, Cancer

\section{Introduction}

Cancer is the leading cause of death of children and adolescents around the world with approximately 300,000 new cases diagnosis each year. In September 2018, the World Health Organization [1] announces the WHO Global Initiative for Childhood Cancer, with the aim of helping countries reaching at least $60 \%$ survival rate for children with cancer by 2030 , through increased prioritization of childhood cancer and an expanded capacity to deliver best practice in childhood cancer care. Children with cancer in low-andmiddle-income countries (LMIC) like Mexico, are four times more likely to die of the disease than children of high-income countries.

Cancer occurs more frequently in adolescents and young adults ages 15 to 39 years than in younger children. The causes of most childhood cancers are unknown [2]. About $5 \%$ of all cancers in children are caused by an inherited genetic mutation (a mutation that can be passed from parents to their children). The diagnosis and treatment of cancer are uniquely traumatic stressors [3] and produce significant 
stress in children and adolescents [4]. A large number of children and adolescents with cancer are victims of traumatic experiences. The most common being bullying in person, cyberbullying; emotional, physical, and/or sexual abuse, including that perpetrated by peers [5].

Eye Movement Desensitization and Reprocessing (EMDR) therapy is guided by the Adaptive Information Processing (AIP) model [6]; which posits that memory networks are the basis of pathology and health. Briefly stated, the AIP is a model of pathogenesis and change. This unique theoretical model posits that psychopathology is primarily caused by memories of adverse life experiences that have been inadequately processed and maladaptively stored in a statespecific form. These memories are stored by association and form memory networks that link present experiences to past experiences and can be triggered by current internal and external stimuli, contributing to present dysfunction. The AIP is the cornerstone of EMDR Therapy because it interprets clinical phenomena, predicts successful treatment outcomes and guides clinical practice. EMDR therapy uses a standardized eight-phase procedure, during which clients focus on elements of the disturbing memory, while simultaneously experiencing bilateral stimulation.

In their AIP model-based Acute Trauma and Ongoing Traumatic Stress Theoretical Conceptualization, Jarero and Artigas [7] assert that from a memory networks perspective (patterns of associated memories), acute trauma situations are related not only to a time frame (days, weeks, or months), but also to a post-trauma safety period $[8,9]$. Their hypothesis is that often, as a result of this ongoing lack of safety, the consolidation of the traumatic memory network is prevented. Therefore, the continuum of external stressful events that creates a network of linked pathogenic memories [10] with similar emotional, somatic, sensorial, and cognitive information, does not give the state-dependent traumatic memory [11] sufficient time to consolidate into an integrated whole. Thus, the memory network remains in a permanent excitatory state as a short-term memory, expanding with each subsequent stressful event to the original adverse experience in this continuum; analogous to the ripple effect of a pebble thrown into a pond, creating a cumulative trauma exposure memory network [12] that extends into the present moment, and often producing maladaptive/catastrophic concerns about the future or flash-forwards [13].

They believe that this type of historical trauma with ongoing traumatic stressors and no post-trauma safety period for memory consolidation requires a different kind of EMDR treatment approach than that used for events which have a post-trauma safety period. Therefore, Jarero et al. [9] adapted the standard EMDR-Integrative Group Treatment Protocol (EMDR-IGTP) for adults [14] to treat adolescents (13 to 17 years old) and adults living with ongoing traumatic stress with no post-trauma safety period for memory consolidation. Changes in the EMDR-IGTP Adapted for Ongoing Traumatic Stress (EMDR-IGTP-OTS) include asking the client to run a mental movie of the whole event from right before the beginning until today, or into the future, and then to identify the hardest, most painful, or most distressing moment. This adaptation was made to encompass the whole traumatic stress spectrum and allow for the identification, targeting, and processing of the continuum of multiple traumatic experiences faced by this population. See Jarero et al. [15] for a detailed description of the EMDR-IGTP-OTS.

Jarero et al. [9] conducted a pilot study to evaluate the effectiveness of EMDR-IGTP-OTS in reducing cancerrelated posttraumatic stress disorder (PTSD) for adult women. EMDR intensive therapy was administered for three consecutive days, twice daily, to 24 adult women diagnosed with different types of cancer (cervical, breast, colon, bladder, and skin) who had PTSD symptoms related to their diagnosis and treatment. Treatment outcomes were compared between patients in the active phase of cancer treatment and those in the follow-up phase, with scores on the Short PTSD Rating Interview (SPRINT) [16] at pre- and post-EMDR treatment and at 30- and 90-day follow-up. Results showed no difference between groups, with significant improvement in both groups for PTSD symptoms and overall subjective well-being.

To extend the previous investigation, Jarero et al. [17] conducted a randomized controlled trial (RCT) on the provision of the EMDR-IGTP-OTS to 65 female adults' patients with different types of cancer and cancer-related PTSD symptoms. A comparison of the treatment and notreatment control groups showed a significantly greater decrease for the treatment group on symptoms of PTSD measured with the Posttraumatic Stress Disorder Checklist for DSM-5 [18] (PCL-5) [19]; anxiety and depression symptoms measured with the Hospital Anxiety and Depression Scale (HADS) [20] in the pre-post and 90-days follow-up measurements, suggesting that the EMDR-IGTPOTS may be an efficient and effective way to address cancerrelated posttraumatic, depressive, and anxious symptoms.

Roberts [21] conducted a pre-experimental case study to explore the efficacy and safety of the EMDR Group Traumatic Episode Protocol (G-TEP) [22] on 35 cancer survivors with various types of cancers in different stages. Participant's received two 90-minutes of G-TEP sessions, administered in consecutive days. Repeated measures comparison of measures of PTSD symptoms, anxiety and depression revealed some significant differences over time and modest changes across the entire sample between posttest and follow-up. Results support the need for research with large samples and randomized clinical trials to examine the viability of providing G-TEP to cancer survivor.

\section{Objective}

The objective of the study was to evaluate the effectiveness of the EMDR-IGTP-OTS in reducing posttraumatic stress disorder symptoms, depression and anxiety symptoms related to the diagnosis and treatment of different types of cancer in adolescents and young adults' patients with cancer. 


\section{Method}

\subsection{Design}

To measure the effect of EMDR-IGTP-OTS on the dependent variables PTSD, Anxiety, and Depression, this single-blind study used a randomized control study design, comparing treatment and no-treatment control groups. Symptoms of PTSD, anxiety, and depression were measured in three-time points: Time 1 pretreatment, Time 2 posttreatment, and Time 3 follow-up.

\subsection{Ethics and Gold Standards}

The research protocol was reviewed and approved by the A New Hope (Una Nueva Esperanza) Center ethics committee and the EMDR Mexico International Research Ethics Review Board (IRERB; also known in the United States as Institutional Review Board), to ensure that the research quality of this study partially fulfilled the Revised Gold Standard scale [23] items. These included: 1) target symptoms were clearly defined, but without diagnosis, 2) measures were reliable and valid, 3) blind independent evaluators collected posttreatment measures at Times 2 and 3 ; 4) assessor reliability was checked by M. G., 5) treatment was manualized, 6) blind random assignment was conducted, 7) treatment fidelity was evaluated by S. G. T, 8) no conditions were confounded, 9) multimodal measures were not used, and 10) length of treatment was appropriate for civilian participants with single trauma (5 or more sessions). Participation was voluntary with a signed informed consent form.

\subsection{Participants}

This study was conducted in 2018 in the city of Puebla, Mexico at the "Una Nueva Esperanza" (A new hope) Center installations. This non-profit organization was founded in 1999 to support children, adolescents and young adults (up to 22 years) with cancer. Potential participants were recruited by explaining the research project during the A New Hope Center monthly reunion to their parents (in case of minors) and young adults. Those who accepted received an appointment for an interview immediately after their next medical appointment in which qualified research assistances explored whether they met or not the inclusion criteria. A total of 42 potential participants participated in the monthly reunion research project explanation, 24 attended the intake interview and 23 fulfill the inclusion criteria. One participant did not meet the inclusion criteria for psychiatric reasons.

Inclusion criteria were: (a) Between 13 and 22 years old or older, (b) diagnosis of cancer, (c) be in the active or followup phase of cancer treatment, (d) with Posttraumatic Stress Disorder Checklist for DSM-5 (PCL-5) symptoms related to their diagnosis and cancer treatment, (e) not receiving specialized trauma therapy, (f) not receiving drug therapy for the posttraumatic stress disorder symptoms.

Exclusion criteria were: (a) ongoing self-harm/suicidal or homicidal ideation, (b) diagnosis of psychotic or bipolar disorder, (c) diagnosis of dissociative disorder, (d) organic mental disorder, (e) current, active chemical dependency problem, (f) significant cognitive impairment (e.g., severe intellectual disability, dementia).

Twenty-three adolescents and young adults (13 male and 10 female) with different types of cancer (breast, leukemia, lymphoma) and PTSD symptoms related to their diagnosis and cancer treatment met the inclusion criteria. Participants age ranged from 13 to 22 years old ( $\mathrm{M}=16.71$ years $)$. Participant's time since diagnosis varied from 2006 to 2018.

Participants were randomly selected to treatment group $(\mathrm{N}=11)$ or no-treatment control group $(\mathrm{N}=12)$ using a computer-generated random-number list. Two independent assessors blind to treatment conditions conducted the randomization process to avoid allocation influence.

Participants were contacted by phone to inform them if they belonged to the treatment group or the no-treatment control group. Patients in the no-treatment control group were informed that for ethical reasons, they would receive the treatment once the first part of the study was concluded. There were 11 participants in the treatment group and 12 participants in the no-treatment control group.

\subsection{Instruments}

1) The Posttraumatic Stress Disorder Checklist for DSM5 [18] (PCL-5) [19] Spanish version provided directly by the National Center for PTSD (NCPTSD) and adapted, with the NCPTD approval, for the past week instead of the past month symptoms to conduct research with high mobility population. It contains 20 items, including three new PTSD symptoms (compared with the PTSD Checklist for DSM-IV) [24]: blame, negative emotions, and reckless or self-destructive behavior. Respondents indicate how much they have been bothered by each PTSD symptom over the past week (rather than the past month), using a 5-point scale ranging from $0=$ not at all, $1=$ a little bit, $2=$ moderately, $3=$ quite a bit and $4=$ extremely. Item scores are summed to yield a continuous measure of PTSD symptom severity for symptom clusters and for the whole disorder. According to the National Center for PTSD, a PCL-5 cut-point of 33 appears to be a reasonable value to propose until further psychometric work is available.

2) The Hospital Anxiety and Depression Scale (HADS) [20] is widely-used to measure psychological morbidity in cancer patients [25]. The HADS is a 14 item self-report scale, using a 4-point Likert scale ranging from 0 to3. Seven items relate to anxiety and seven to depression. For this study, we used the Spanish adaptation of the HADS for patients with cancer [26]. This adaptation showed good internal consistency and validity. An alpha coefficient of .85 and a split-half reliability of .8 were found. Cut-off points of 8 and 9 for the anxiety and depression subscales, respectively, showed a favorable sensitivity and specificity in identifying cases of psychological disorder as defined by the psychiatric diagnosis using the DSM-IV-TR [24]. 


\subsection{Procedure}

Treatment and no-treatment control group participants completed the instruments on individual bases in the different measurement moments. By Time 1, research assistants collected clinical histories, provided psycho-education and answered patient's or parent's questions related to trauma, PTSD, and EMDR therapy to decrease any possible prejudice against the treatment. Application of instruments for both groups was done after this procedure by other research assistants who were not blind to the study but blind to the treatment allocation. During Time 2 (post-treatment assessment) and Time 3 (follow-up assessment 90 days after treatment) assessment was conducted for all participants by blind independent assessors with a master's degree in clinical psychology who were not part of the study. Data was collected, stored, and handled in full compliance with the EMDR Mexico IRERB requirements to ensure confidentiality. It is important to mention that in the different measurement moments all participants were asked to focus specifically on the worst cancer-related event that currently bothered them the most before answering the PCL-5.

In this study, intensive EMDR therapy [27, 28] was provided because many participants traveled to the A New Hope Center from long distances and only stayed there for a limited time. This intensive format allowed patients to complete the full course of treatment in a short period. EMDR-IGTP-OTS was administered by six licensed EMDR clinicians formally trained in the protocol administration. One was a certified EMDR therapist, and five were certified EMDR therapists in training.

The treatment group participants received six treatment sessions during two consecutive days, three times daily in a setting inside the A New Hope Center where they received cancer treatment or follow-up. EMDR-IGTP-OTS treatment focused only on the trauma memories related to the oncological disease and did not address any previous traumatic events. The first group session lasted 1 hour and 46 minutes. The next group sessions had an average duration of 53 minutes since they started in Phase 3 of the protocol, as it was not necessary to repeat Phases 1 and 2. Participants received an average of 6 hours and 18 minutes treatment in total. There were no dropouts. No adverse effects were reported during treatment or at 90-day follow-up. Treatment fidelity and adherence to the protocol was fulfilled by EMDR therapists' strict observance to all steps of the scripted protocol 15] while they were assessed by the supervisor (S. G. T).

\section{Statistical Analysis}

Analyses of variance (ANOVA) for repeated measurements was used with three-time points for PTSD, anxiety, and depression. Analyses included two groups (treatment $11=$ subjects; no-treatment control $=12$ subjects) as independent variables and PTSD, anxiety, and depression as dependent variables. Results of the beta square variables are presented. Cohen's $d$ was calculated to report the size of the significant effects of the t-test comparisons.

\section{Results}

PTSD (PCL-5). Data analysis by repeated measures ANOVA revealed a significant effect for time $(F(2,42)=$ $17.02 \mathrm{p}<.001, \eta_{P}^{2}=.448$ ), a significant effect for group (F $\left(1,21=5.57, \mathrm{p}<.05, \eta_{P}{ }^{2}=.210\right)$ and a significant interaction between time and group, $\left(\mathrm{F}(2,42)=22.18, \mathrm{p}<.001, \eta_{P}{ }^{2}\right.$ $=.513)$. In the treatment group, mean scores showed a significant decrease between time 1 and time $2, \mathrm{t}(10)=4.03$, $\mathrm{p}<.005, d=.75$. There was also a significant decrease between time 1 to time $3, \mathrm{t}(10)=5.25, \mathrm{p}<.001, d=1.17$. In the control group no significant differences were found between means in all comparisons. Comparison between groups did not show significant differences for time 1. For time 2 and 3 significant differences among the groups were founded, $\mathrm{t}(21)=-2.63, \mathrm{p}<.01, d=.77$ and $\mathrm{t}(21)=-4.38, \mathrm{p}$ $<.01, d=1.30$ respectively. See Table 1 and figure 1 .

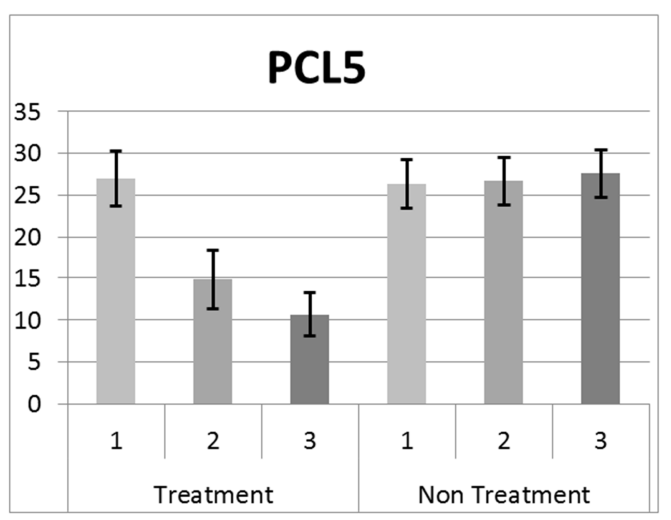

Figure 1. Mean scores and standard error for PCL-5 by time and group.

Table 1. Mean scores (M) and standard deviations (SD) for treatment and no-treatment group on pretest, posttest and follow up measurements.

\begin{tabular}{|c|c|c|c|c|c|c|}
\hline & \multicolumn{2}{|l|}{ Time 1} & \multicolumn{2}{|l|}{ Time 2} & \multicolumn{2}{|c|}{ Time 3} \\
\hline & $\bar{M}$ & SD & $\mathbf{M}$ & SD & $\mathbf{M}$ & SD \\
\hline \multicolumn{7}{|c|}{ Posttraumatic Stress (PCL-5) } \\
\hline Treatment group & 27.00 & 11.02 & 14.81 & 31.77 & 10.63 & 8.54 \\
\hline $\begin{array}{l}\text { No treatment group } \\
\text { Anxiety }\end{array}$ & 26.33 & 10.21 & 26.66 & 9.72 & 27.58 & 9.84 \\
\hline Treatment group & & 5.99 & 3.45 & 2.58 & 2.81 & 1.77 \\
\hline No treatment group & 6.75 & 5.01 & 6.50 & 5.46 & 5.25 & 4.57 \\
\hline \multicolumn{7}{|l|}{ Depression } \\
\hline Treatment group & 5.45 & 3.14 & 2.72 & 2.96 & 2.09 & 2.50 \\
\hline No treatment group & 4.33 & 3.98 & 4.25 & 4.90 & 5.41 & 4.87 \\
\hline
\end{tabular}


Anxiety. Repeated measures analysis of variance (ANOVA) showed a significant effect for group $(\mathrm{F}(2,42)=$ $\left.10.64, \mathrm{p}<.001, \eta_{P}^{2}=.336\right)$ and a significant interaction effect between time and group $(\mathrm{F}(2,42)=19.77, \mathrm{p}<.05$, $\left.\eta_{P}{ }^{2}=.163\right)$. Significant differences between time 1 and time 2 were found for the treatment group, $\mathrm{t}(10)=2.49, \mathrm{p}<.05, d$ $=.78$, and between time 1 and time $3, \mathrm{t}(10)=3.70, \mathrm{p}<.005, d$ $=99$. No significant differences were observed in the no treatment control group between different time application. Comparison between groups did not show significant differences for this variable. See Table 1 and figure 2.

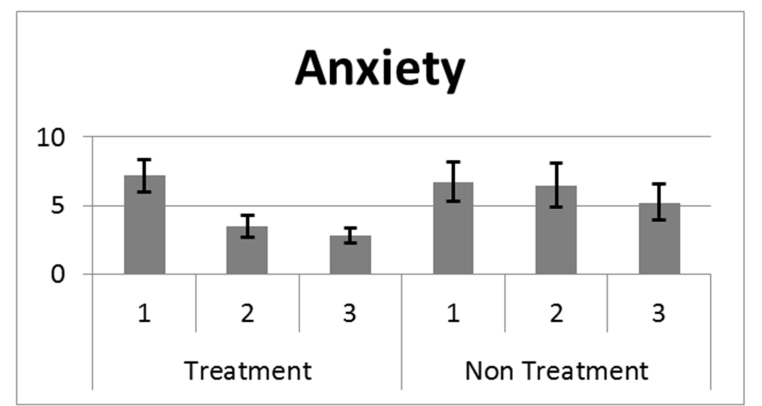

Figure 2. Mean scores and standard error for Anxiety by time and group.

Depression. Results showed significant interaction effects between time and group. $\left(\mathrm{F}(2,42)=6.49, \mathrm{p}<.005, \eta_{P}^{2}\right.$ $=.236$ ). For the treatment group significant differences were found between time 1 and time $2, \mathrm{t}(10)=2.23, \mathrm{p}<.05$, $d=.63)$, and between time 1 and time $3, \mathrm{t}(10)=3.04, \mathrm{p}<.01$, $d=.83$. In the control group, no significant differences were found between means in all comparisons. Comparison between groups showed significant differences for time $3, \mathrm{t}$ $(21)=-2.02, p<.05, d=49$. See Table 1 and figure 3 .

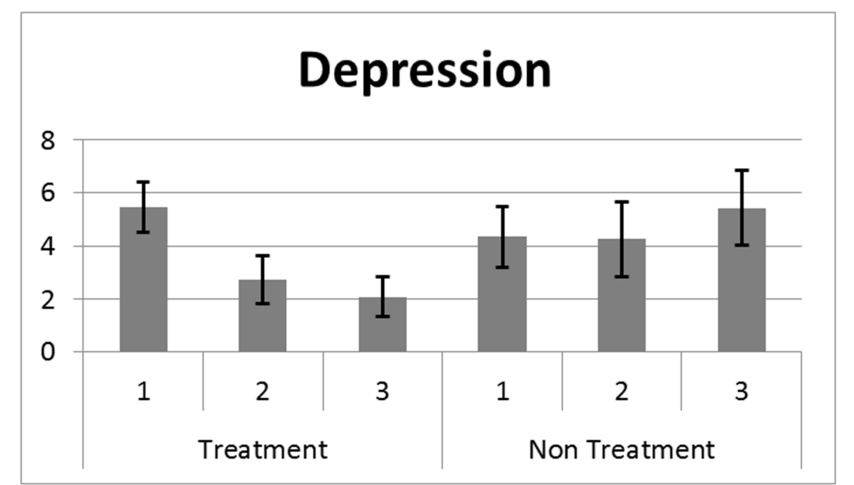

Figure 3. Mean scores and standard error for Depression by time and group.

\section{Discussion}

For PTSD symptoms, the results revealed significant changes through time for the treatment group. The changes were significant between the pretest and the posttest as well as between the posttest and the follow-up. In the control group, the score remained at the same level of the pretest, the posttest, and the follow-up. The effects are showing that the treatment decreases the PTSD symptoms which continue decreasing even after treatment finished.

Concerning anxiety and depression, there were significant differences between the treatment group and the no-treatment control at follow-up. Anxiety decreased significantly from pretest to follow-up in the treatment group, and no changes were observed for the no-treatment control group. Depression decreased significantly in the treatment group from pretest to follow up and increased significantly in the no-treatment control group.

Adolescents and young adults with cancer presented elevated rates of psychological distress (anxiety, depression and posttraumatic stress symptoms) [29]. There is evidence that if left untreated, PTSD in children and young people can lead a chronic course lasting a number of years [30]. To Faretta and Civilotti [31] the application of EMDR therapy could break the vicious cycle between psychological and physical health provoked by cancer-related traumas.

For the adolescents and young adults' population, the EMDR-IGTP-OTS offered advantages because it's minimally intrusive and does not require creating a narrative of the traumatic experience, verbal or written disclosure of details, prolonged reliving traumatic experiences, or homework. Relying on drawings or symbols presents a special advantage to provide culturally sensitive and effective treatment for patients who struggle to connect to their cognitive states or feel guilty or ashamed [17]. These patients may be more comfortable expressing their emotional distress through drawing. Also, drawings are used for effective reprocessing with patients with lower levels of literacy [32] like the participants in this study.

\section{Conclusion}

Experiencing cancer means a peculiar stressor within the infrastructure of PTSD because it involves a potentially chronic and acute debilitating disease. This experience can be accompanied by a wide range of associated adverse events, such as tumor detection, diagnosis, severity of disease and prognosis; aggressive treatment; disfigurement and bodily dysfunction; side effects of treatment; impaired physical, social, and occupational functioning; and sometimes recurrence and diagnosis of terminal illness. Cancer represents an internal and external stressor that is extended over time. Hence, people diagnosed with cancer may be at risk of developing PTSD, anxiety and depression symptoms that adversely impact functionality and genera well-being and may lead to worse survival outcome. Therefore, the implementation of EMDR therapy protocols specially designed for acute trauma and/or ongoing traumatic stress aimed at taking care of psychopathology reported by patients with cancer is an area of fundamental interest. This study results indicate that the intensive administration of the EMDR-IGTP-OTS could be an efficient and effective way to address cancer-related PTSD, depressive, and anxious symptoms in adolescents and young adults. 


\section{Limitations}

The sample size of the study is a limitation. Further research with randomized controlled studies is recommended to replicate these results with a larger sample size of adolescents and young adults with cancer-relates PTSD, anxiety and depression symptoms.

\section{Acknowledgements}

This study was not founded by any grants.

We want to express gratitude to the following individuals for their contributions:

Francisco Álvarez and Laura Arizpe. Alfaro, Luisa Andrea; Álvarez, Elizabeth; Badillo, Claudia; Conde, Rebeca Adriana; De los Santos, Maritza; Domínguez, Carolina; Ehlinger, Paula; Encinas, Mónica; Gómez, Alicia; Millán, María José; Nuño, María Amparo; Ortiz, Gisele; Peregrina, María Alicia; Picazo, María Leticia; Priante, Carmen María; Ramírez, Luis Edgar; Ramírez, Silvia Patricia; Rodríguez, Marine; Romano, María de las Nieves; Ros, Jessica María; Treviño, Andrea.

\section{Conflict of Interest}

All the authors do not have any possible conflicts of interest.

\section{References}

[1] World Health Organization (2018). WHO Global Initiative for Childhood Cancer. Retrieved from http://www.who.int/cancer/en/.

[2] National Cancer Institute (2017). Cancer in Children and Adolescents. National Cancer Institute. Retrieved from: https://www.cancer.gov/types/childhood-cancers/childadolescent-cancers-fact-sheet.

[3] Andrykowski, M. A., Kangas, M. (2010). Posttraumatic stress disorder associated with cancer diagnosis and treatment. 2nd ed. In: Holland JC, Breitbart WS, Jacobsen PB, Lederberg MS, Loscalzo MJ, Mc Corklen R, editors. Oxford Textbook of PsychoOncology. New York: Oxford University Press. p. 348-57.

[4] Bruce, E.; Desjardins, Leandra; Vannatta, Kathryn; YoungSaleme Tammi; Rodriguez, Erin M.; Dunn, Madeleine; Bemis, Heather; Snyder, Sarah; Gerhardt, Cynthia A. (2014). Children and adolescents coping with cancer: Self- and parent reports of coping and anxiety/depression. Health Psychology, 33(8), 853861. Retrieved from: http://psycnet.apa.org/buy/2014-30548-003.

[5] Serrano-Ibáñez, Elena R.; Ruiz-Párraga, Gema T.; Esteve, Rosa; Ramírez-Maestre, Carmen; López Martínez, Alicia E. (2018). Validation of the child PTSD symptom scale in spanish adolescents. Colegio Oficial de Psicólogos del Principado de Asturias, España. http://www.redalyc.org/articulo.oa?id=72754594020.

[6] Shapiro, F. (2018). Eye movements desensitization and reprocessing. Basic principles, protocols, and procedures (Third edition). Guilford Press.

[7] Jarero, I., \& Artigas, L. (2018). AIP model-based Acute Trauma and Ongoing Traumatic Stress Theoretical
Conceptualization. Iberoamerican Journal of Psychotraumatology and Dissociation, 10(1), 1-7.

[8] Jarero, I., Artigas, L., \& Luber, M. (2011). The EMDR protocol for recent critical incidents: Application in a disaster mental health continuum of care context. Journal of EMDR Practice and Research, 5(3), 82-94.

[9] Jarero, I., Artigas, L., Uribe, S., García, L. E., Cavazos, M. A., \& Givaudan, M. (2015). Pilot research study on the provision of the EMDR integrative group treatment protocol with female cancer patients. Journal of EMDR Practice and Research, 9(2), 98-105.

[10] Centonze, D., Siracusane, A., Calabresi, P., and Bernardi, G. (2005). Removing pathogenic memories. Mol. Neurobiol. 32, 123-132.

[11] van der Kolk, B. A., \& van der Hart, O. (1991). The intrusive past: The flexibility of memory and the engraving of trauma. American Imago, 48(4), 425-454.

[12] Jarero, I., Amaya, C., Givaudan, M., Miranda, A. (2013). EMDR Individual protocol for paraprofessional use: A randomized controlled trial with first responders. Journal of EMDR Practice and Research, 7(2), 55-64.

[13] Logie, R., \& de Jongh, A. (2014). The "flashforward" procedure: Confronting the catastrophe. Journal of EMDR Practice and Research, 8, 25-32.

[14] Jarero, I., \& Artigas, L. (2014). The EMDR Integrative Group Treatment Protocol (IGTP) for adults. In M. Luber (Ed.), Implementing EMDR early mental health interventions for man-made and natural disasters (pp. 253-265). New York, NY: Springer Publishing.

[15] Jarero, I., Artigas, L., Uribe, S., \& García, L. E. (2016). The EMDR Integrative Group Treatment Protocol for Patients with Cancer. Journal of EMDR Practice and Research, 10(3), 199-207.

[16] Connor, K. M., \& Davidson, J. R. (2001). SPRINT: A brief global assessment of post-traumatic stress disorder. International Clinical Psychopharmacology, 16(5), 279-284.

[17] Jarero, I., Givaudan, M., Osorio, A. Randomized Controlled Trial on the Provision of the EMDR Integrative Group Treatment Protocol Adapted for Ongoing Traumatic Stress to Female Patients with Cancer-Related Posttraumatic Stress Disorder Symptoms. (2018). Journal of EMDR Practice and Research, 12(3), 94-104.

[18] American Psychiatric Association. (2013). Diagnostic and statistical manual of mental disorders $\left(5^{\text {th }} \mathrm{ed}\right.$.). Arlington, VA.

[19] Weathers, F. W., Litz, B. T., Keane, T. M., Palmieri, P. A., Marx, B. P., \& Schnurr, P. P. (2013). The PTSD Checklist for DSM-5 (PCL-5). Scale available from the National Center for PTSD at www.ptsd.va.gov.

[20] Zigmond, A. S. \& Snaith, R. P. (1983). The Hospital Anxiety and Depression Scale. Acta Psychiatrica Scandinavica, 67, 361-370.

[21] Roberts, A. (2018). The effects of the EMDR Group Traumatic Episode Protocol with cancer survivors. Journal of EMDR Practice and Research, 12(3), 105-117.

[22] Shapiro, E. "The EMDR Group Traumatic Episode Protocol." (2013). Presentation to the EMDR Turkey Conference, Istanbul, Turkey. 
[23] Maxfield, L., \& Hyer, L. (2002). The relationship between efficacy and methodology in studies investigating EMDR treatment of PTSD. Journal of Clinical Psychology, 58, 23-41.

[24] American Psychiatric Association. (2000). Diagnostic and statistical manual of mental disorders ( $4^{\text {th }}$ ed. Text Revised). Arlington, VA.

[25] Ornelas-Mejorada, R. E., Tufiño, M. A., Sánchez-Sosa, J. J. (2011). Ansiedad y depresión en mujeres con cáncer de mama en radioterapia: Prevalencia y factores asociados. Acta de Investigación Psicológica, 1(3), 401-414.

[26] Rico, J. L., Restrepo, M., Molina, M. (2005). Adaptación y validación de la escala hospitalaria de ansiedad y depresión (HAD) en una muestra de pacientes con cáncer del Instituto Nacional de Cancerología de Colombia. Avances en Medición, 3, 73-86.

[27] Bongaerts, H., Van Minnen, A., de Jongh, A. (2017). Intensive EMDR to treat patients with complex posttraumatic stress disorder: A case series. Journal of EMDR Practice and Research, 11(2), 84-95.

[28] Lobenstine, F., \& Courtney, D. (2013). A case study: The integration of intensive EMDR and ego state therapy to treat comorbid posttraumatic stress disorder, depression, and anxiety. Journal of EMDR Practice and Research, 7, 66-80.

[29] McCarthy, M. C., McNeil, R., Drew, S., Dunt, D., Kosola, S., Orme, L., Sawyer, S. M. (2016). Psychological Distress and Posttraumatic Stress Symptoms in Adolescents and Young Adults with Cancer and Their Parents. Journal of Adolescents and Young Adults Oncology, 5(4), 59-64.

[30] Yule W, Bolton D, Udwin O, Boyle S, O'Ryan D, Nurrish J. (2000). The long-term psychological effects of a disaster experienced in adolescence: I: The incidence and course of PTSD. J Child Psychol Psychiatry. 41: 503-11.

[31] Faretta, E., \& Civilotti, C. (2016). EMDR therapy in psychooncology: A bridge between mind and body. Journal of EMDR Practice and Research, 10(3), 138-152.

[32] Shapiro, F. Clinician's Corner: EMDR Therapy. International Society for Traumatic Stress Studies. Stress Points. (2016). Retrieved from http://www.istss.org/educationresearch/traumatic-stresspoints/2016-april/clinician-s-corneremdr-therapy.aspx. 